\title{
Ethical challenges in mental health research with focus in Nepal
}

Pratik Khanal $^{1,3^{*}}$, Sajana Maharjan ${ }^{2}$

${ }^{1}$ Global Health Unit, Institute of Medicine, Kathmandu, Nepal

${ }^{2}$ Nepal Health Research Council, Kathmandu, Nepal

${ }^{3}$ Karma Health, Kathmandu, Nepal

Received:

28 August 2017

Revised:

13 November 2017

Accepted:

16 January 2018

\begin{abstract}
People with mental health problems are considered as vulnerable population by national ethical guidelines of health research in Nepal. There are different ethical challenges in research involving people with mental health problems. Ethical challenges are related to study design, autonomy, beneficence and nonmaleficence, justice, respect for the environment and consent taking process. Respecting the human rights of those with mental health problems is necessary during research and this requires research stakeholders to be responsible.
\end{abstract}

Keywords: ethical challenges, mental health, Nepal, research

\author{
${ }^{\star}$ Corresponding author \\ pratikkhanal@iom.edu. \\ np \\ Global Health Unit, Institute of \\ Medicine
}

Tweetable Abstract: Mental health research are surrounded by ethical challenges. Research stakeholders need to be responsible while conducting mental health research.

\section{Introduction}

Research plays a crucial role in shaping health policy and decision making. The benefits that the research brings however come under expenses of the research participants whose contributions are usually undermined during the research process. Ethical issues often surround health research and are of special concern when vulnerable populations like those with mental health problems are involved [1]. Research in mental health like any other research should follow ethical principles and should be guided by national ethical guidelines.

Health research in Nepal is governed by Nepal Health Research Council (NHRC) established in 1991 and guided by National Ethical Guidelines for Health Research in Nepal and Standard Operating Procedures 2011 [2]. This paper discusses three major areas related to research ethics in mental health research in Nepal. Firstly, we have listed provisions mentioned in national ethical guidelines related to mental health research. Secondly, we have tried to explore the ethical challenges that may occur during conduction of mental health research and thirdly, we have elucidated the responsibilities of different stakeholders involved in mental health research. The paper draws on experiences of the authors as internal reviewers during their work in ethics section of NHRC.

\section{Provision regarding mental health in national ethical guidelines}

The national ethical guidelines for health research in Nepal 2011 [2] has defined people with mental disabilities as a vulnerable group in research and has mentioned two conditions for involving mentally ill patients in the study. The first is when such research cannot be carried out satisfactorily in person in full possession of their mental faculties and the other condition is when the purpose of the research is to obtain knowledge relevant to the health needs of people with mental disorders. Thus, the study should have a clear rationale for involving participants with a mental disorder.

One major issue that arises while involving participants with mental disorder is of informed consent. Are the participants able to understand the study objective and provide informed consent and if not how do we protect their autonomy? The guidelines [2] mention that assent should be taken to the extent possible from the persons with mental disorders and informed consent be taken from their legal guardian. In case of inability to give personal consent, the inclusion of people with mental health disorders in clinical trial is acceptable only when the ethics committee and the investigator thinks that participation will promote the health and welfare of the research participants and the agreement made by a legal guardian is documented [3]. The clinical trial guidelines of Nepal 2005 [3] further states that every precaution should be taken to respect the privacy of the research participants and to minimize the impact of the study on the participant's physical and mental integrity and on their personality. 


\section{Ethical challenges in mental health research}

Ethical challenges in mental health research are usually related to study design, autonomy of the research participants, benefits, justice, respect for the environment and consent taking process [4-6]. We here briefly discuss the ethical challenges under different headings:

\section{a. Study design}

Study design such as research involving placebos where the patient might be refrained from standard treatment or efficacy proved drugs, washout designs in which there might be discontinuation of medications and symptom provocation design where the individuals might be exposed to behavioral or medical response would be harmful to the persons with mental illness [7]. People with mental health problems should only be involved in research which contributes to the health and well-being of the research participants. This however requires strong scientific reasoning and the protocol needs to be pertinent to established research regulations [6].

The data collection tool employed in mental health research may provoke sentiments among research participants which lead to the unnecessary recall of the memories related to tragic or unexpected events. Research with children investigating behaviors such as emotional and behavioral problems, suicidal tendency, drug abuse, and deviancy related problems will be ethically challenging [8]. Situations as such demand a competent researcher/research team who can deal with psychosocial issues and can manage the unexpected events resulting from the research process. The study design thus should involve ethically appropriate framework and protect human participants from undue harm or influence.

\section{b. Autonomy}

Respect for the autonomy of persons is a well-established ethical principle. However, during research, research participants may not be in a position to provide informed consent as mental disorders may compromise their cognitive ability. It is often difficult to categorize what type of people with mental disorders can provide informed consent and who cannot. Researcher needs to be sure that the study participants comprehend the purpose of the research which is often challenging in case of people with mental health disorders. It is thus necessary that those with diminished autonomy be protected against harm or abuses. Usually, assent needs to be obtained from the participants and consent be taken from close relatives and caregivers [9]. Assent is necessary because being mentally ill is not similar to being incompetent to make decisions about themselves [10].

\section{c. Beneficence and Nonmaleficence}

People suffering from mental health problems may not be competent enough to weigh the risks and benefits of the study. This condition of the unequal relationship between researchers and research participants might increase the vulnerability of research participants. The study population of mental health research also includes healthy population or populations who have recently been affected by disaster or trauma. Research at times of such tragic events needs to be carefully designed and the ethics committees need to carefully review such protocol so that participants may not be exposed to additional harms[5]. Research should be evaluated in terms of the benefits it brings to the body of knowledge and to the population involved in the study or similar population outside research.

\section{d. Justice}

In any research, it is necessary to consider whether the research is of benefit to the research participants. On the one hand, the research should not put the already vulnerable population exposed to more risks and on the other hand, mental health patients should benefit from the advancement of research and cure. Thus, it is necessary that research in mental health be critically evaluated on the perspectives of equity and social justice. Special provisions need to be made for the protections of rights and welfare of the research participants in vulnerable situations. This includes situations when the participants are unable to protect their interests due to lack of capacity to consent or do not have alternative means of medical care.

\section{e. Respect for the environment}

It is likely that participants with mental health problems are exposed to undue medical influences because of their condition. It is thus necessary to treat with respect the biological and genetic heritage of these people involved in the research. Equally important is maintaining the privacy and confidentiality of the data collected for the research. Like any other research, it is also necessary that social, cultural and natural heritage of societies and communities be protected. In communities where there is low literacy or where there is low research familiarity, it is often recommended to consider community trust before approaching the community households [11].

\section{f. Consent taking process}

Informed consent is often seen by researchers as a bureaucratic trail but it has to be seen as a process of recognition of the research participants in the broader community. It is often difficult for the researchers to decide about how much information to share with the research participants in mental health research as full disclosure might affect the outcome of the study [9]. The conditions as such must be fully explained in the protocol. Every chance should be provided to the research participants to be fully informed about the research process and the options for voluntary participation and withdrawal at any time. The consent taken either fully informed consent or assent should be documented.

\section{Stakeholders' responsibility in maintaining research ethics}

There are different stakeholders involved in the research process. It is however the researcher or the research team who play a prominent 
role in adhering to specific ethics requirement of research ethics committees and promoting the human rights of the research participants.

\section{a. Responsibility of researcher}

Researcher needs to make sure that the protocol has been approved by the research ethics committee. It is the responsibility of the researcher to disclose the source of funding and share interim and final progress report to the ethics committee and report any required amendments in the protocol. It is the duty of the researcher to make sure that research participants are empowered enough to participate in the research process and data collection procedures are safe and pose no harmful risks [6]. The results of the research should also be communicated to the research participants.

If the study is being done in general population and mental disorders have been diagnosed during the study, it becomes the responsibility of the researcher to communicate it to the participants and provide necessary treatment or referral support.

\section{b. Responsibility of reviewer}

A reviewer needs to maintain professional integrity and consider ethical principles while reviewing the protocol. Reviewer should declare a conflict of interest if any while reviewing the proposal and should put the research participants at the center during review. A reviewer needs to be competent and should send an unbiased review of the research proposals to the ethics committee. It is usually the review sent by the reviewers that form the basis of decision making by the ethics committee.

\section{c. Responsibility of research ethics committee}

Research ethics committees are responsible to carefully review the protocol and make decisions based on different criteria. The criteria are usually the risk of study in relation to the benefits and the knowledge it contributes, fair selection of the study population, data collection method, informed consent taking process and maintenance of privacy and confidentiality. In case of trials or research involving risks, ethics committee need to make sure that research team has the plan to minimize the risk, manage the adverse events if any and have provision for referral and insurance[3]. Research ethics committees are responsible not only to approve or disapprove the research protocol but also monitor the progress of the research project. Special care should be taken by the ethics committee in research involving vulnerable population including people with mental health problems.

\section{d. Responsibility of research participants}

Research participants need to carefully understand and evaluate the information provided by the research team regarding the research process. They have the right to make further inquiry to the research team about the purpose, methodology, procedures involved, anticipated risks and benefits and their role in research. However, research participants in Low and Middle-Income Countries easily provide consent due to low research literacy and are influenced by health workers, incentives, and free clinical services [12]. It is the responsibility of the research community including civil societies and government bodies to make sure that research participants are not harmed by the research activities and are best benefited by the advancements in research.

\section{Conclusion and Way Forward}

National ethical guidelines have identified people with mental health disorders as a vulnerable group. Thus, it is the role of all stakeholders that human rights of this population be protected and they are benefited by the advancements in clinical care. In the context of increment of research in area of mental health in Nepal mostly by non-government organizations[13] (NHRC received 26 research proposals related to mental health research in 2014, 38 in 2015 and 72 in 2016) [14], we hope the following recommendations which are also supported by literature would be useful to promote research ethics in relation to mental health research in Nepal and elsewhere.

a) Researcher needs to be trained in research ethics, communication skills and in understanding the perspectives of the research participants about the research. $[9,11,15]$ This can be done by incorporating research ethics in university curricula and training of researchers. [16].

b) The national guidelines need to be revised to help researchers to deal with vulnerable populations including people with mental disorders. The consent taking process, the legal age for informed consent and special precautions such as during clinical trials and disasters need to be of focus in the guidelines.

c) Local community and those organizations or forums involved in mental health need to be involved during protocol development and approval should be taken from the local ethics committee to gain trust with research participants and understand the social context in which research takes place $[4,17]$.

d) Academia, research organizations and mental health policy makers and program managers need to come together to define research priorities in mental health to promote evidence informed mental health policy and services. This will help to guide research and funding agencies to focus on areas where mental health research is needed.

\section{References}

1. Slowther, A., P. Boynton, and S. Shaw, Research governance: ethical issues. Journal of the Royal Society of Medicine, 2006. 99(2): p. 65-72.

2. NHRC, National Ethical Guidelines for Health Research in Nepal and Standard Operating Procedures. 2011, Nepal Health Research Council: Kathmandu.

3. NHRC, National Guidelines on Clinical Trials with the Use of Pharmaceutical Products 2005, Nepal Health Research Council: Kathmandu. 
4. Chiumento, A., et al., Managing Ethical Challenges to Mental Health Research in Post-Conflict Settings. Developing world bioethics, 2016. 16(1): p. 15-28.

5. Siriwardhana, C., et al., Ethical challenges in mental health research among internally displaced people: ethical theory and research implementation. BMC medical ethics, 2013. 14(1): p. 13.

6. Roberts, L.W., C.M. Geppert, and J.L. Brody, A framework for considering the ethical aspects of psychiatric research protocols. Comprehensive psychiatry, 2001. 42(5): p. 351-363.

7. DuVal, G., Ethics in psychiatric research: study design issues. The Canadian Journal of Psychiatry, 2004. 49(1): p. 55-59.

8. Hiriscau, E.I., et al., Identifying ethical issues in mental health research with minors adolescents: results of a Delphi study. International journal of environmental research and public health, 2016. 13(5): p. 489.

9. Fulford, K. and K. Howse, Ethics of research with psychiatric patients: principles, problems and the primary responsibilities of researchers. Journal of Medical Ethics, 1993. 19(2): p. 85-91.

10. Lidz, C.W., Can People with Mental Illness Consent to Research? Psychiatry Information in Brief, 2006. 3(1): p. 1.

11. Mohamed, A. and D. Loewenthal, Is it possible to ethically research the mental health needs of the Somali communities in the UK. 2009.

12. Regmi, P.R., et al., Informed Consent in Health Research: Challenges and Barriers in Low-and Middle-Income Countries with Specific Reference to Nepal. Developing world bioethics, 2017. 17(2): p. 84-89.

13. Upadhaya, N., et al., The role of mental health and psychosocial support nongovernmental organisations: reflections from post conflict Nepal. Intervention, 2014. 12: p. 113-128.

14. NHRC. Ethics data by area. 2017 [cited 201701 July, 2017]; Available from: http://nhrc.gov.np/ethics/.

15. Upadhaya, N., et al., Current situations and future directions for mental health system governance in Nepal: findings from a qualitative study. International journal of mental health systems, 2017. 11(1): p. 37.

16. Adhikari, S., et al., Knowledge, attitude and practice of healthcare ethics among resident doctors and ward nurses from a resource poor setting, Nepal. BMC medical ethics, 2016. 17(1): p. 68.

17. DuBois, J., et al., Ethical issues in mental health. Current opinion in psychiatry, 2011. 24(3): p. 208. 